\title{
Oral Contraceptives and Cardiovascular Risk: Adding Clinical Evidence to the Pathophysiology
}

\author{
Daniel Arthur B. Kasal ${ }^{1,2}$ and Andrea De Lorenzo ${ }^{10}$ \\ Instituto Nacional de Cardiologia, ${ }^{1}$ Rio de Janeiro, RJ - Brazil \\ Universidade do Estado do Rio de Janeiro, ${ }^{2}$ Rio de Janeiro, $R J$ - Brazil
}

Women cardiovascular health is an important and often neglected issue. Cardiovascular diseases (CVDs) are the main cause of death in women, in Brazil and worldwide. ${ }^{1}$ Oral contraceptives are the main method used for contraception in Brazil. ${ }^{2}$ The issue of oral contraceptives and cardiovascular risk has been raised since the first descriptions of this class of pharmaceuticals, in the 1960s. The development of low-dose combined oral contraceptives (COCs) containing ethinyl estradiol and different progestins has reduced, albeit not eliminated, cardiovascular morbidity in women taking these medications. ${ }^{3}$ The main side effects associated with COCs are procoagulant effects. Therefore, the use of COCs is associated with increased risk of developing acute myocardial infarction, venous thromboembolism and stroke. ${ }^{4}$ In addition, adverse changes in the lipid profile and glucose tolerance have been described. ${ }^{5}$ Clearly, ageing and exposure to other risk factors, mainly smoking and obesity, play an important role in the development of adverse outcomes related to COCs. In addition, formulations with different progestins may produce distinct effects on circulation. ${ }^{6}$

Another important side effect associated with COCs is the development of hypertension. A meta-analysis including 24 studies and over 250,000 participants found a significant association between the duration of oral contraceptive use and risk of hypertension. ${ }^{7}$ Studies regarding the pathophysiology of this association have revealed a number of different and complementary mechanisms. These include oxidative stress ${ }^{8}$ endothelial

\section{Keywords}

Oral Contraceptives/adverse effects; Blood Coagulation Factors; Oxidative Stress/drug effects; Hypertension; Woman; Morbidity. dysfunction and the activation of the Renin-AngiotensinAldosterone system (RAAS). ${ }^{9}$ In this issue, Oliveira et al., ${ }^{10}$ published an interesting clinical observational study, with young volunteers using low-dose COCs for at least one year, compared to women not using the medication. The authors found higher plasma renin levels in women using COC, with a strong correlation with plasma C-reactive protein (CRP). Additionally, although they did not reach the level of hypertension, systolic arterial pressure measurements of COC users were higher than those of non-users. This finding suggests that RAAS activation, if sustained, could eventually shift blood pressure in women using this medication into the hypertension range. The increase of inflammatory pathways, which is associated with RAAS activation, is also shown by the positive correlation with CRP. While the study was restricted to young and healthy participants, and COC use included five different formulations, the results add clinical evidence linking RAAS activation to oral contraceptive use, inflammation and even hypertension development, in a Brazilian sample.

As in all cross-sectional studies, the results cannot establish a cause-effect relationship between COC and CRP elevation or increased blood pressure, but rather suggest an association between them and generate hypotheses. Additionally, it is not possible to exclude that other factors might have influenced the RAAS balance, such as diet, as the authors have already pointed out.

The complexity of the RAAS - with its multiple regulatory pathways - makes its study a challenging task. Nonetheless, the present results of Oliveira et al. ${ }^{10}$ highlight the need to thoroughly evaluate cardiovascular risk factors and perform adequate cardiovascular physical examination in patients planning to use $\mathrm{COC}$; and to properly exert regular clinical follow-up of these individuals, aiming at the early diagnosis of adverse effects, such as blood pressure elevation.

Mailing Address: Daniel Arthur B. Kasal

Av. 28 de Setembro, 77 - $3^{\circ}$ andar, sala 329. Postal Code: 20550-900, Rio de Janeiro, RJ - Brazil

E-mail: dabkasal@gmail.com, andlorenzo@hotmail.com

DOI: https://doi.org/10.36660/ijcs.20200078 


\section{References}

1. Brasil. Ministério da Saúde. Saúde Brasil 2018 uma análise de situação de saúde e das doenças e agravos crônicos: desafios e perspectivas. Brasília; 2019.

2. Farias MR, Leite SN, Tavares NUL, Oliveira MA, Arrais PS, Bertoldi AD et al. et al. Use and access to oral and injectable contraceptives in Brazil. Rev Saude Publica 2016; 50:14s.

3. Torgrimson BN, Meendering JR, Kaplan PF, Minson CT. Endothelial function across an oral contraceptive cycle in women using levonorgestrel and ethinyl estradiol. Am J Physiol Heart Circ Physiol. 2007; 292(6):H2874-80.

4. Lalude OO. Risk of cardiovascular events with hormonal contraception: insights from the Danish cohort study. Curr Cardiol Rep. 2013;15(7):374.

5. Shufelt CL, Bairey Merz CN. Contraceptive hormone use and cardiovascular disease. J Am Coll Cardiol. 2009;53(3):221-31.
6. Rosendaal FR, Helmerhorst FM, Vandenbroucke JP. Female hormones and thrombosis. Arterioscler Thromb Vasc Biol. 2002;22:201-10.

7. Liu H, Yao J, Wang W,Zhang D. Association between duration of oral contraceptive use and risk of hypertension: A meta-analysis. J Clin Hypertens (Greenwich). 2017;19(10):1032-41.

8. Chen JT, Kotani K. Oral contraceptive therapy increases oxidative stress in pre-menopausal women. Int J Prev Med. 2012;3(12):893-6.

9. Olatunji LA, Seok YM, Igunnu A. Combined oral contraceptive-induced hypertension is accompanied by endothelial dysfunction and upregulated intrarenal angiotensin II type 1 receptor gene expression. Naunyn Schmiedebergs Arch Pharmacol. 2016;389(1):14\&-57. 389(1):1147-57.

10. Oliveira SS, Petto J, Diogo DP, Santos AC, Sacramento MS, Ladeia AMT, et al. Plasma Renin in Women Using and Not Using Combined Oral Contraceptive. Int J Cardiovasc Sci. 2020;33(3):208-214. 